\title{
Pneumocystis jirovecii pneumonia in an HIV-infected patient mimicking acute eosinophilic pneumonia: a case report with a review of the literature
}

\author{
Hiroshi Ohji $^{1}$, Tsutomu Shinohara ${ }^{2}$, Naoki Kadota ${ }^{1}$, Yoshio Okano ${ }^{1}$, Keishi Naruse ${ }^{3}$, Yoshihito Iwahara ${ }^{4}$, \\ Fumitaka Ogushi ${ }^{1}$ \\ ${ }^{1}$ Division of Pulmonary Medicine, ${ }^{2}$ Department of Clinical Investigation, ${ }^{3}$ Division of Pathology, ${ }^{4}$ Division of Hematology, National Hospital \\ Organization Kochi Hospital, Kochi, Japan \\ Correspondence to: Tsutomu Shinohara. Department of Clinical Investigation, National Hospital Organization Kochi Hospital, 1-2-25 \\ Asakuranishimachi, Kochi 780-8077, Japan. Email: shinohara.tsutomu.kg@mail.hosp.go.jp.
}

Submitted May 29, 2018. Accepted for publication Sep 29, 2018.

doi: $10.21037 /$ jtd.2018.10.49

View this article at: http://dx.doi.org/10.21037/jtd.2018.10.49

\section{Introduction}

Pneumocystis jirovecii pneumonia (PCP) is the most common opportunistic infection with the highest lethality among acquired immunodeficiency syndrome (AIDS)-related diseases, and there are many cases where HIV infection is revealed by PCP as an initial manifestation. In Japan, AIDS patients unaware of their HIV status account for approximately $30 \%$ of newly diagnosed HIV-positive patients, and the percentage of PCP is the highest at approximately $30 \%$ among AIDSindicator diseases $(1,2)$. Pneumocystis jirovecii interacts with many kinds of host cells in the lung and induces lung injury. Although onset of HIV-positive PCP is usually slow, the clinical presentation including respiratory failure occasionally takes an acute course. The typical chest radiographic finding of PCP is ground-glass opacity (GGO), sometimes accompanied by nodular shadows and/or consolidations $(3,4)$. Therefore, differential diagnosis from various diffuse lung diseases, including progressive interstitial pneumonia, is not always easy. In addition, eosinophilia (greater than or equal to 5 percent) in bronchoalveolar lavage (BAL) fluid (BALF) has been observed in about $20 \%$ of HIV-positive PCP patients (5).

Acute eosinophilic pneumonia (AEP) is a disease concept proposed by Allen et al. in 1989 and characterized by fever, diffuse pulmonary infiltrates (GGO with or without airspace consolidation) and BALF eosinophilia (greater than 25 percent) in an acute course, leading to a high rate of respiratory failure $(6,7)$. Although AEP is idiopathic, it is favorable for young adult males and a relationship with smoking has been reported in a number of cases. Thus, AEP is presumed to be an acute hypersensitivity reaction to some inhaled antigen and, in general, steroid therapy is effective (6).

In this manuscript, we report a HIV-positive PCP case with prominent BALF eosinophilia (38.5\%), which was initially treated as AEP, and discuss the usefulness of serum and BALF biomarkers for pneumonitis, such as serum surfactant protein D (SP-D) and Krebs von den Lungen-6 (KL-6), in the diagnosis of this condition.

\section{Case presentation}

A 44-year-old man in previous good health was admitted to our hospital due to a 1-week history of dyspnea and fever without severe fatigue, muscle weakness or weight loss. The patient had no clinical history of sexually transmitted disease or fungal infection, and had not received any medication. He smoked ten cigarettes a day for 1 year about 20 years earlier. Physical examination revealed slightly decreased breath sounds in both lungs. Laboratory data were as follows: white blood cell count, $6,880 / \mu \mathrm{L}$ [neutrophils, $59.7 \%$; lymphocytes, $32.4 \%\left(2.2 \times 10^{3} / \mathrm{L}\right)$; eosinophils, $2.6 \%$; monocytes, $0.0 \%$; basophils, $0.4 \%$ ]; hemoglobin, $15.1 \mathrm{~g} / \mathrm{dL}$; platelet count, $14.0 \times 10^{4} / \mu \mathrm{L}$; C-reactive protein, 0.98 mg/dL; SP-D, $90.1[<107$ (8)] ng/mL; serum KL-6, $2,330[<476(8)] \mathrm{U} / \mathrm{mL}$. Mild hypoxemia $\left(\mathrm{SpO}_{2} 94 \%\right.$ on room air) was noted, but there were no other signs of HIV or opportunistic infection, such as LDH elevation 

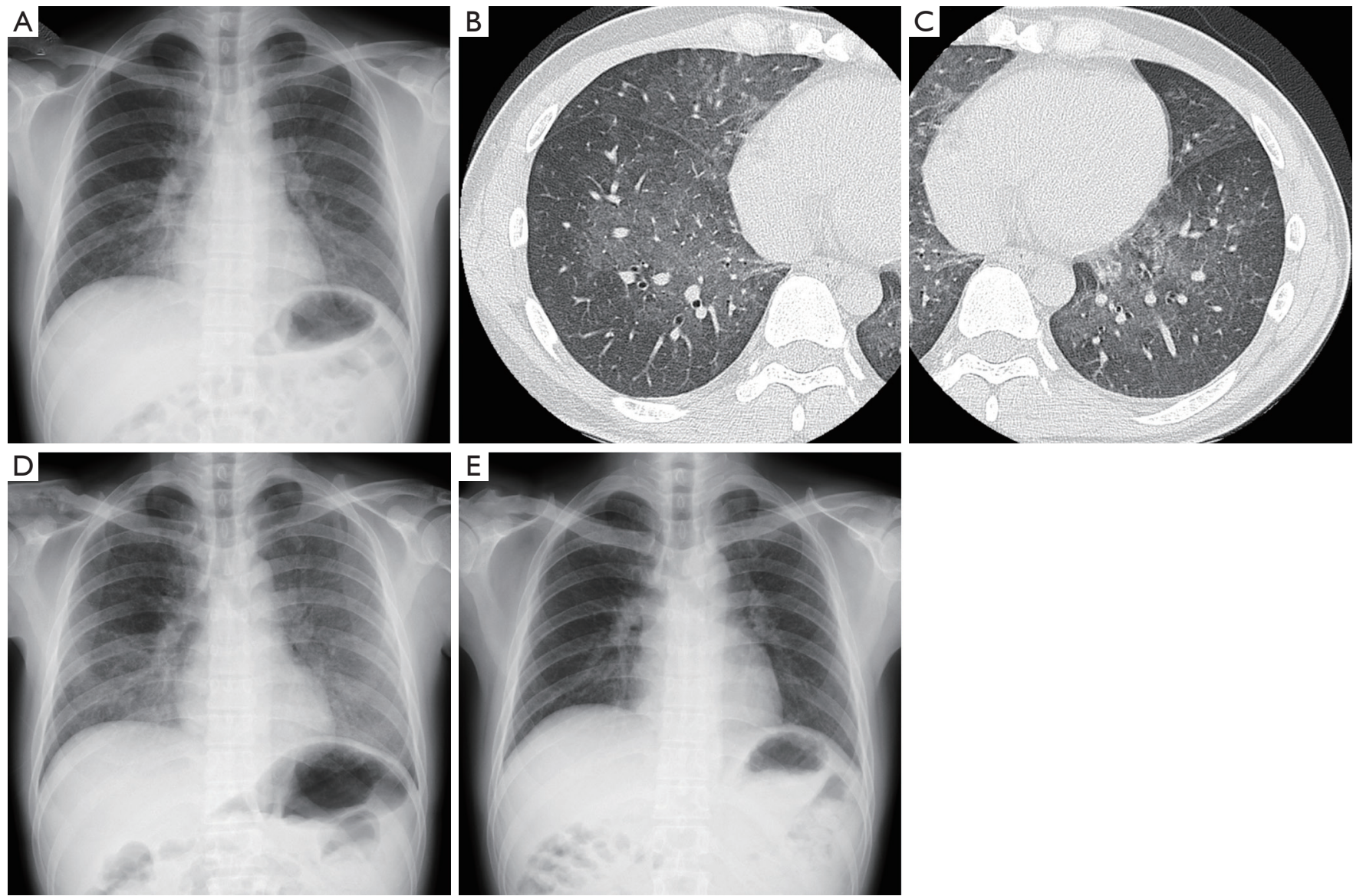

Figure 1 Chest X-ray (A, D and E) and high-resolution CT (B and C) findings. (A,B,C) Bilateral ground-glass opacities (GGOs) without chronic changes on admission to hospital; (D) worsening of pre-existing GGOs two weeks after the initiation of treatment using oral prednisolone (30 mg/day); (E) marked improvement after 3 months of treatment (administration of trimethoprim-sulfamethoxazole, atovaquone and ganciclovir followed by anti-HIV therapy).

and liver disorder. Chest X-ray and high-resolution CT showed bilateral GGOs without chronic changes (Figure 1). Bronchoscopic examination was performed on suspicion of interstitial pneumonia and BAL (recovery rate; $64 / 150)$ revealed high total cellularity $\left(42 \times 10^{4}\right.$ cells $\left./ \mathrm{mL}\right)$ consisting of $38.5 \%$ eosinophils, $37.5 \%$ neutrophils, $16.5 \%$ lymphocytes and $7.5 \%$ alveolar macrophages. BALF smear and culture showed normal flora and transbronchial lung biopsy (TBLB) detected no specific findings. AEP was clinically suspected and the patient was treated with oral prednisolone $(30 \mathrm{mg} /$ day $)$. However, his respiratory condition worsened $\left(\mathrm{SpO}_{2} 92 \%\right.$ at rest receiving oxygen via a non-rebreather mask at $12 \mathrm{~L} / \mathrm{min}$ ) and GGOs clearly progressed over two weeks (Figure 1). Methylprednisolone pulse therapy (1,000 $\mathrm{mg}$ intravenously for 3 days) was added. Then, a highly elevated $(1 \rightarrow 3)-\beta-D$-glucan (BDG) level
[185 pg/mL: cut-off level was $20 \mathrm{pg} / \mathrm{mL}$ (9)] in the serum was observed. Additional sectioning of the TBLB specimen revealed eosinophilic foamy areas on hematoxylin and eosin staining and accumulation of black-colored cysts on Grocott methenamine silver stain in alveolar lesion (Figure $2 A, B$ ). No findings suggesting cytomegalovirus pneumonia, such as nuclear inclusion bodies, were observed in the lung tissue. Polymerase chain reaction (PCR) analysis of sputum was positive for Pneumocystis jirovecii. The serum anti-HIV-1 antibody was positive and peripheral blood CD4-positive lymphocytes decreased to $18 / \mathrm{L}$. HIV-1 western blot analysis was positive and real-time PCR assay revealed a HIV-1 RNA of $7.3 \times 10^{4}$ copy $/ \mathrm{mL}$. Cytomegalovirus (CMV) antigenemia was positive. Additional examination of BALF showed that the concentrations of BDG, SP-D and KL-6 were $1,010 \mathrm{pg} / \mathrm{mL}, 804 \mathrm{ng} / \mathrm{mL}$ and 5,626 U/mL respectively 

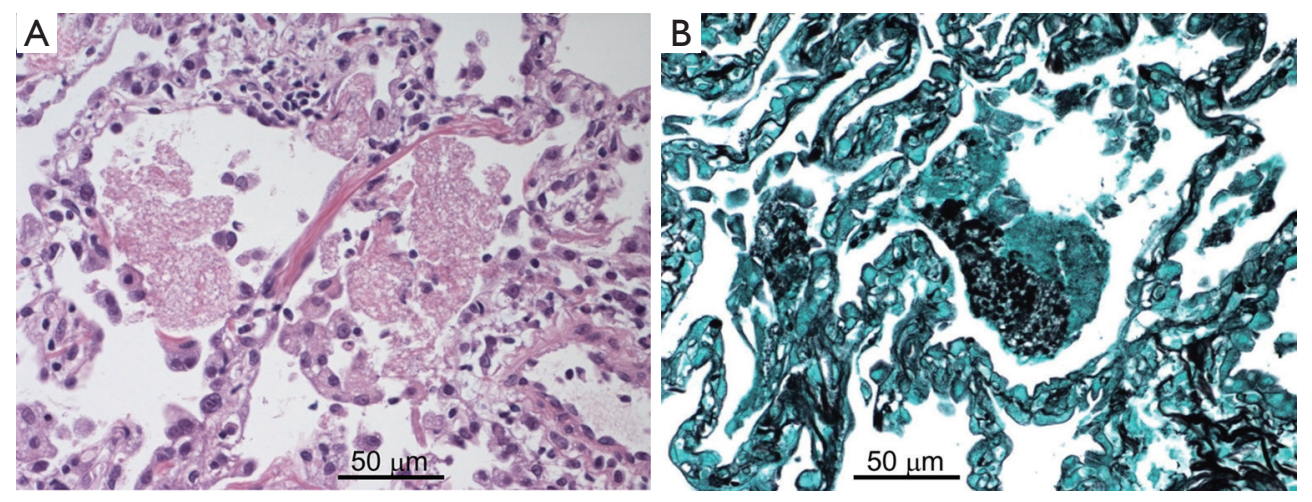

Figure 2 Pathological findings of transbronchial lung biopsy specimens (A, hematoxylin and eosin staining; B, Grocott methenamine silver staining). (A) Eosinophilic foamy areas in alveolar lesions; (B) accumulation of black-colored cysts.

[cut-off levels of BALF BDG and SP-D were not described previously $(10,11)$, but that of KL-6 was 340 (12)]. PCP and CMV infection complicated with AIDS was diagnosed. Trimethoprim-sulfamethoxazole, atovaquone and ganciclovir were administered followed by anti-HIV therapy (raltegravir and emtricitabine/tenofovir disoproxil fumarate). Hypoxemia and abnormal findings on chest $\mathrm{X}$-ray improved after 3 months of this treatment (Figure 1).

\section{Discussion}

HIV-positive PCP occasionally resembles AEP in terms of clinical symptoms, chest radiographic findings and BALF cell classification. Both diseases may progress rapidly and cause severe respiratory failure, but the treatments for each disease are fundamentally different and prompt differentiation is important.

There are many PCP patients who do not know that they are infected with HIV and these patients do not always have accompanying peripheral blood lymphopenia. It was reported that peripheral blood absolute lymphocyte count (ALC) was $1.1 \times 10^{3} / \mu \mathrm{L}$ or more in $25 \%$ of cases where HIV infection was revealed by PCP and, as observed in our patient, ALC in $7.5 \%$ of the cases was more than $2.0 \times 10^{3} / \mathrm{L}$ (13). Generally, distribution of GGO shows central predominance in HIV-positive PCP, whereas in AEP it shows peripheral predominance. However, in half the patients with each disease, the distribution does not meet this trend, and consolidations, interlobular septal thickening and nodules are common findings in both diseases $(3,7)$. These reports suggest that further insight into differentiation between HIV-positive PCP and AEP is necessary.
BDG is a major constituent of the cell wall in pneumocystis jirovecii, and it was reported that both serum and BALF BDG levels are elevated in HIV-positive PCP (Table 1), as shown in our case $(10,15,16)$. However, even in HIV-positive PCP cases, no increase in concentration of serum BDG was observed in about $5 \%$ of cases $(10,15,16)$. On the other hand, Kawayama et al. reported that BDG did not increase in serum in AEP, but rose in BALF (Table 1). The authors considered the possibility that BDG inhaled from the environment directly acted as a biological response modifier and induced eosinophilic inflammation in the alveolus (11). Thus, it is not always easy to distinguish between HIV-positive PCP and AEP with only BALF and serum BDG values.

In cases where serum BDG has not yet been measured or is in the normal range and in whom bronchoscopic examination has not been performed, one differential diagnosis of HIV-positive PCP or AEP is progressive interstitial pneumonia and, in routine practice, evaluation of markers for pneumonitis such as SP-D and KL-6 is usually done. SP-D is a glycoprotein mainly produced in type II alveolar epithelial cells and is directly secreted into the alveolar spaces. On pulmonary injury, the efflux of SP-D from damaged epithelial secretory cells into the alveoli and its sift into the blood due to the increased permeability of lung vessels induce the elevated serum SP-D level (19). On the other hand, KL-6 is a cell membrane constituent glycoprotein with a huge molecular weight, and mainly expressed in type II alveolar epithelial cells and bronchiolar epithelium. When alveolar epithelium is strongly injured due to fibrosing interstitial pneumonia etc., the production of KL-6 by regenerating type II alveolar epithelial cells is enhanced, and KL-6 cleaved just outside the cell membrane 
Table 1 BDG, SP-D and KL-6 levels in the serum/BALF of an HIV-positive PCP and AEP case

\begin{tabular}{|c|c|c|c|c|}
\hline Group & Sample & BDG level (pg/mL) & SP-D level (ng/mL) & KL-6 level (U/mL) \\
\hline \multirow[t]{2}{*}{ Control } & Serum & $<20(9)^{\|}$ & $<107(8)^{\text {ๆ }}$ & $<476(8)^{9}$ \\
\hline & BALF & $2.5 \pm 1.4^{*}(11)^{\pi}$ & $196 \pm 2.4^{*}(14)^{\natural}$ & $<340(12), 195 \pm 2.4^{*}(14)^{\natural}$ \\
\hline \multirow[t]{3}{*}{ HIV-positive PCP } & Serum & $494.1[8.5-1,135]^{\dagger}(10)$ & & \\
\hline & & $300[32-4,822]^{\ddagger}(15)$ & & $1,120[331-4,330]^{\ddagger}(15)$ \\
\hline & BALF & $7,268[1,355-15,500]^{\dagger}(10)$ & & \\
\hline \multirow[t]{2}{*}{ AEP } & Serum & $4.4 \pm 6.0[1.0-16.5]^{\S}(11)$ & $261 \pm 104[138-416]^{\S}(14)$ & $164 \pm 34[112-205]^{\S}(14)$ \\
\hline & BALF & $851.7 \pm 866.0[216-2,571]^{\S}(11)$ & $1,693 \pm 2,233^{*}(14)$ & $161 \pm 74^{*}(14)$ \\
\hline \multicolumn{5}{|c|}{ HIV-positive PCP mimicking AEP (BALF eosinophil \%) } \\
\hline Case $1(20.6 \%)(17)$ & Serum & 210 & & 429 \\
\hline Case $2(15.0 \%)(18)$ & Serum & 114 & & 4,260 \\
\hline Case $3(56.0 \%)(18)$ & Serum & 99 & & 812 \\
\hline
\end{tabular}

${ }^{*}$, mean $\pm \mathrm{SD} ;{ }^{\dagger}$, mean [range]; ${ }^{\ddagger}$, median [range]; ${ }^{\S}$, mean $\pm \mathrm{SD}$ [range]; ${ }^{\prime \prime}$, patients without fungal infection; ", healthy control. BDG, $(1 \rightarrow 3)-\beta-D-g l u c a n ;$ SP-D, surfactant protein D; KL-6, Krebs von den Lungen-6; BALF, bronchoalveolar lavage fluid; PCP, Pneumocystis jirovecii pneumonia; AEP, acute eosinophilic pneumonia.

accumulate in the alveolar space. Then KL-6 transfers to the blood like SP-D (20).

Daimon et al. reported that serum and BALF SP-D levels were elevated in AEP, but KL-6 levels were within normal range (Table 1) (14). These data probably reflect the fact that there is no strong alveolar epithelial injury in AEP. In contrast, a clinical study revealed that the concentration of serum KL-6 was high in HIV-positive PCP, probably reflecting epithelial injury, although BALF KL-6 was not measured (Table 1) (15). In this case, KL-6 was elevated in both BALF and serum, suggesting that KL-6 is a marker for HIV-positive PCP even in cases showing prominent BALF eosinophilia. An elevated level of SP-D was observed only in BALF but not in serum, the reasons for which are unclear at this time. However, up-regulation of BALF SP-D may reflect a host defense mechanism enhancing opsonization in interactions between Pneumocystis jirovecii and alveolar macrophages rather than an extensive type II epithelial cells injury (21). To the best of our knowledge, there are three reported cases of HIV-positive PCP mimicking AEP, two of which indicated an elevated serum level of KL-6. However, the significance was not discussed (Table 1) $(17,18)$. The accumulation of similar cases is necessary to evaluate the specificity and sensitivity of KL-6 as a biomarker of HIVpositive PCP with prominent BALF eosinophilia.

\section{Conclusions}

For patients who present with an acute course with GGO, prominent BALF eosinophilia, and elevated BALF and/or serum KL-6 level(s), we need to consider HIV-positive PCP, even when peripheral blood lymphopenia is not observed.

\section{Acknowledgements}

We would like to thank Mikio Miyauchi (Division of Clinical Laboratory, National Hospital Organization Kochi Hospital) for his contribution.

\section{Footnote}

Conflicts of Interest: The authors have no conflicts of interest to declare.

Informed Consent: Written informed consent was obtained from the patient for publication of this manuscript and any 
accompanying images.

\section{References}

1. Ogawa T, Shirasaka T, Imamura T. Review and Estimation of "Detection Rate of AIDS Patients without Knowing HIV Status (Ikinari-AIDS Rate)". The Journal of AIDS Research 2012;14:46-54.

2. Yanagawa Y. Clinical Perspective - Pneumocystis Pneumonia. Jpn J Chest Dis 2014;73:1040-56.

3. Tasaka S. Pneumocystis Pneumonia in Human Immunodeficiency Virus-infected Adults and Adolescents: Current Concepts and Future Directions. Clin Med Insights Circ Respir Pulm Med 2015;9:19-28.

4. Boonsarngsuk V, Sirilak S, Kiatboonsri S. Acute respiratory failure due to Pneumocystis pneumonia: outcome and prognostic factors. Int J Infect Dis 2009;13:59-66.

5. Smith RL, el-Sadr WM, Lewis ML. Correlation of bronchoalveolar lavage cell populations with clinical severity of Pneumocystis carinii pneumonia. Chest 1988;93:60-4.

6. Allen JN, Pacht ER, Gadek JE, et al. Acute eosinophilic pneumonia as a reversible cause of noninfectious respiratory failure. N Engl J Med 1989;321:569-74.

7. Daimon T, Johkoh T, Sumikawa H, et al. Acute eosinophilic pneumonia: Thin-section CT findings in 29 patients. Eur J Radiol 2008;65:462-7.

8. Hamai $\mathrm{K}$, Iwamoto $\mathrm{H}$, Ishikawa $\mathrm{N}$, et al. Comparative Study of Circulating MMP-7, CCL18, KL-6, SP-A, and SP-D as Disease Markers of Idiopathic Pulmonary Fibrosis. Dis Markers 2016;2016:4759040.

9. Obayashi T, Yoshida M, Mori T, et al. Plasma ( $1 \rightarrow 3)-\beta-D-$ glucan measurement in diagnosis of invasive deep mycosis and fungal febrile episodes. Lancet 1995;345:17-20.

10. Yasuoka A, Tachikawa N, Shimada K, et al. $(1 \rightarrow 3)$ $\beta$-D-glucan as a quantitative serological marker for Pneumocystis carinii pneumonia. Clin Diagn Lab Immunol 1996;3:197-9.

Cite this article as: Ohji H, Shinohara T, Kadota N, Okano Y, Naruse K, Iwahara Y, Ogushi F. Pneumocystis jirovecii pneumonia in an HIV-infected patient mimicking acute eosinophilic pneumonia: a case report with a review of the literature. J Thorac Dis 2018;10(11):E774-E778. doi: 10.21037/ jtd.2018.10.49
11. Kawayama T, Fujiki R, Honda J, et al. High concentration of $(1 \rightarrow 3)-\beta$-D-glucan in BAL fluid in patients with acute eosinophilic pneumonia. Chest 2003;123:1302-7.

12. Kohno N, Awaya Y, Oyama T, et al. KL-6, a mucinlike glycoprotein, in bronchoalveolar lavage fluid from patients with interstitial lung disease. Am Rev Respir Dis 1993;148:637-42.

13. Omene AA, Ferguson RP. Absolute lymphocyte count as a predictor of Pneumocystis pneumonia in patients previously unknown to have HIV. J Community Hosp Intern Med Perspect 2012;2(1).

14. Daimon T, Tajima S, Oshikawa K, et al. KL-6 and surfactant proteins $\mathrm{A}$ and $\mathrm{D}$ in serum and bronchoalveolar lavage fluid in patients with acute eosinophilic pneumonia. Intern Med 2005;44:811-7.

15. Nakamura H, Tateyama $M$, Tasato $D$, et al. Clinical utility of serum beta-D-glucan and KL-6 levels in Pneumocystis jirovecii pneumonia. Intern Med 2009;48:195-202.

16. Watanabe T, Yasuoka A, Tanuma J, et al. Serum $(1 \rightarrow 3)$ $\beta$-D-glucan as a noninvasive adjunct marker for the diagnosis of Pneumocystis pneumonia in patients with AIDS. Clin Infect Dis 2009;49:1128-31.

17. Itoh $M$, Nakamura H, Nemoto K, et al. A case of AIDScomplicated lung infection mimicking acute eosinophilic pneumonia. Nihon Kokyuki Gakkai Zasshi 2006;44:589-94.

18. Yamauchi S, Hatanaka S, Nishida M, et al. Two cases of bronchoalveolar lavage eosinophilia associated with Pneumocystis pneumonia. Kokyū 2006;25:908-11.

19. Hartl D, Griese M. Surfactant protein D in human lung diseases. Eur J Clin Invest 2006;36:423-35.

20. Ishikawa N, Hattori N, Yokoyama A, et al. Utility of KL-6/MUC1 in the clinical management of interstitial lung diseases. Respir Investig 2012;50:3-13.

21. Atochina EN, Beck JM, Scanlon ST, et al. Pneumocystis carinii pneumonia alters expression and distribution of lung collectins SP-A and SP-D. J Lab Clin Med 2001;137:429-39. 